\title{
Evaluation of anaesthetic techniques for surgery of upper extremities
}

\author{
Koj Jarbo ${ }^{1}$, Keya Chakraborty ${ }^{2 *}$, Dhrubajyoti Sarkar ${ }^{3}$, Pratap B Kaushik ${ }^{4}$
}

\author{
1,3Senior Specialist, Professor and Head, Department of Anaesthesiology, Tomo Riba Institute of Health and Medical Science (TRIHMS), \\ Naharlagun, Arunachal Pradesh, INDIA. \\ ${ }^{2}$ Assistant Professor, Department of Anaesthesiology, Ramakrishna Mission Seva Pratisthan and Vivekananda Institute of Medical Sciences, \\ Kolkata, West Bengal, India \\ ${ }^{4}$ Lecturer-Statistics, Department of PSM, RNT Medical College, Udaipur, Rajasthan, India. \\ Email: drkeyachakraborty2114@gmail.com
}

Abstract Background: The anaesthetic technique used in surgeries of upper extremities is determined by factors such as urgency, presence of coexisting health problems, preference of patient, availability of equipments and drugs, preference and experience of the anaesthetist and surgeon. Methods: This is a retrospective study of all the upper extremity surgeries requiring anaesthesia services that occurred in the period between 1st October, 2014 to 30th September, 2019 in the department of Anaesthesiology, TRIHMS, Naharlagun, Arunachal Pradesh, India. The anaesthesia techniques used for surgeries of upper extremities were evaluated in this study. Anaesthesia methods were recorded as general anaesthesia (GA) and regional anaesthesia (RA). General anaesthsia was classified into GA with Endotracheal Intubation, GA with LMA and GA with Face Mask and RA was classified into Brachial Plexus block (Inter-scalene, Supraclavicular, Infraclavicular or Axillary), Intravenous Regional Anaesthesia ( Bier Block ) and Distal Nerve Block subgroups. RA groups were further categorized into those performed with and without the use of aids such as Ultrsound or Nerve stimulator. Results: During the study period, a total of 771 patients, 498 male and 273 female, underwent surgeries for upper extremity. The commonest site operated upon was forearm. The most frequently employed anaesthetic technique during the total study period was general anaesthesia (73.8\%). In the first 3 years of the study period, all the patients received GA $(100 \%)$, and in the 5th year, the usage of RA jumped to $175(67.56 \%)$. All the RAs were performed with the aid of Ultrasound. The most commonly performed RA was Supraclavicular Brachial Plexus Block. Conclusions: In recent years, regional anaesthesia administration in surgery of upper extremities is gradually increasing, and Supraclavicular brachial plexus block is the most frequently performed regional anaesthesia. There may be a need to explore the use of other forms of regional anaesthesia.

Keywords: Upper extremity surgery, General anaesthesia, Regional anaesthesia, USG-guided nerve block.

*Address for Correspondence:

Dr. Keya Chakraborty, Assistant Professor, Department of Anaesthesiology, Ramakrishna Mission Seva Pratisthan and Vivekananda Institute of Medical Sciences, Kolkata, West Bengal, INDIA.

Email: drkeyachakraborty2114@gmail.com

Received Date: 12/11/2019 Revised Date: 10/12/2019 Accepted Date: 26/01/2020

DOI: https://doi.org/10.26611/10151321

\begin{tabular}{|l|l|}
\hline \multicolumn{2}{|c|}{ Access this article online } \\
\hline Quick Response Code: & Website: \\
\hline & www.medpulse.in \\
\cline { 2 - 2 } & \\
\hline
\end{tabular}

\section{INTRODUCTION}

General Anaesthesia (GA) and Regional Anaesthesia (RA) have been successfully used for upper extremity surgeries $^{1,2}$. In the past, general anaesthesia was frequently the method of choice for upper extremity surgery due to lack of training and experience in the field of regional anaesthesia as well as fear of complications including vascular puncture, local anaesthetic toxicity, pneumothorax, and patient discomfort ${ }^{3}$. However, the advantages of GA, including control of airway and the familiarity of the technique by the majority of anaesthesiologists are overshadowed by clear benefits of regional anaesthesia, which includes intra operative as

\footnotetext{
How to site this article: Koj Jarbo, Keya Chakraborty, Dhrubajyoti Sarkar, Pratap B Kaushik. Evaluation of anaesthetic techniques for surgery of upper extremities. MedPulse International Journal of Anesthesiology. February 2020; 13(2): 36-40.

http://medpulse.in/Anesthsiology/index.php
} 
well as postoperative analgesia, excellent muscle relaxation during surgery, decreased opioid requirements, greater haemodynamic stability, increased efficiency in the operating room, decreased Postoperative Nausea and Vomiting (PONV), reduced Post Anaesthetic Care Unit (PACU) stay, decrease in unplanned hospital admission for pain control, as well as greater patient satisfaction ${ }^{4,5}$. RA is particularly desirable and effective in elderly and high-risk patients undergoing upper and lower extremity surgeries ${ }^{6}$. The use of electrical nerve stimulation to locate peripheral nerves was introduced in $1962{ }^{7}$. Several advantages have been attributed to this technique, including a higher success rate, the avoidance of vascular injury and paresthesias, and associated neurological injuries ${ }^{8-10}$. The use of a Peripheral Nerve Stimulator (PNS) has been the 'gold standard' for performing peripheral nerve blocks for the last two decades. However, with the recent developments in high-frequency imaging, the use of Ultrasound (US) technology has significantly increased for nerve localization ${ }^{11,12}$. The US-guided technique offers reported additional advantages, including avoidance of intraneural/intravascular injection, decrease in dose and volume of local anaesthetics, faster onset time, improved block quality, decreased pain from muscular contractions, prolonged postoperative analgesia, and decreased need for rescue analgesics ${ }^{13-15}$. In this study, we compiled and evaluated the various kinds of anaesthetic techniques practiced for upper extremity surgeries in the past 5 years at Tomo Riba Institute of Health and Medical Sciences (TRIHMS), Arunachal Pradesh, India.

\section{METHODS}

After obtaining approval from the institutional ethics committee, all the patients who underwent upper extremity surgeries during the study period were included. This is a retrospective study of all the anaesthetic technique performed for upper extremity surgeries over a period of 5 year period between 1st October, 2014 to 30st September, 2019 in the department of Anaesthesiology, TRIHMS Hospital, Naharlagun, Arunachal Pradesh, India. Data were collected and analyzed from the Operation Theatre Record Books. Data collected included the age and sex of the patients, indication and the surgery performed. Anaesthesia techniques were recorded as GA, RA, and combined RA with GA. GA was further classified into GA with Endotracheal intubation (ETT), GA with LMA and GA with face mask, RA was further classified into Interscalene Brachial plexus block (ISB), Supraclavicular Brachial plexus block (SCBPB), Axillary Brachial plexus block (AXBPB), Suprascapular nerve block (SSNB), wrist block (WB), Radial and Ulnar nerve block (RUNB) and Intravenous Regional Anaesthesia (Bier Block).
Regional Anaesthesia groups were further categorized into those performed with and without the use of aids such as Ultrasound or Nerve stimulator. The various anaesthetic techniques used for upper extremity surgeries were grouped and recorded year wise for evaluation. Descriptive statistics were presented as patient number and percentages $(\%)$ for nominal variables. Statistical analysis has been done using EPI INFO-7 Software and by chi-squared test, $p$-value of less than 0.05 is considered to be significant.

\section{RESULTS}

During the study period, a total of 771 patients 498 male and 273 female, underwent upper extremity surgeries: 99, $117,128,168$ and 259 patients in the first, second, third, fourth and fifth year of the study period respectively. The demographic profile of the patients according to age and sex are as shown in the Figure-1. Analysis shows that the maximum number of patients belonged to the age group of 21-30 years of age $231(29.96 \%)$ and the males (65.1\%), with a $\mathrm{M}: \mathrm{F}=1.86: 1$, outnumbered females across almost all age groups. The distribution of cases as per the indication for and site of surgeries are as shown in Figure-2. Analysis shows that forearm was the most frequently operated site $440(57 \%)$ followed by shoulder $116(15 \%)$. Of the total $771,569(73.8 \%)$ patients received GA, $184(23.86 \%)$ patients received RA or distal nerve blocks, and $18(2.33 \%)$ patients received combined GA/Sedation and RA. The year wise breakdown of the anaesthetic techniques employed (Figure. 3 and Table 1) shows that in the first 3 years of the study period all the patients were given GA, maximum being the GA with endotracheal tube intubation and the least being GA with LMA. In the year 2017-18, of the total of 168 patients, $141(83.93 \%), 20(11.9 \%)$, and $7(4.2 \%)$ patients received GA, RA and RA combined with GA respectively. And, in the year 2018-19, of the 259 patients who underwent upper extremity surgeries, only $84(32.43 \%)$ patients were provided GA, whereas, $163(62.93 \%)$ patients received $\mathrm{RA}$ in the form of Brachial Plexus Block (ISB, SCBPB, AXBPB, SSNB, WB) and $1(0.38 \%)$ patient was given distal nerve block (RUNB). The remaining 11(4.24\%) patients also received RA, but they required supplementation with Sedation/GA. Among the RA techniques the most frequently performed RA is Supraclavicular Brachial Plexus Block, which comprised $19(70.37 \%)$ in the year 2017-18 and $138(78.85 \%)$ in the year 2018-19. All the RA were performed under sonoguidance. It has been observed that in the last two years, the number of cases gradually increased and there is a significant ( $p$-value $<0.001$ ) increase in the practice of regional anaesthesia with decrease in the practice of General anaesthesia (Figure-4 and Table-2). 


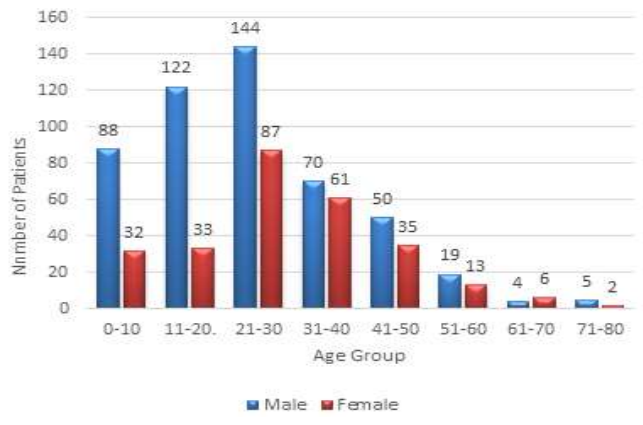

Figure 1: Distribution of Patients According to Age and Sex

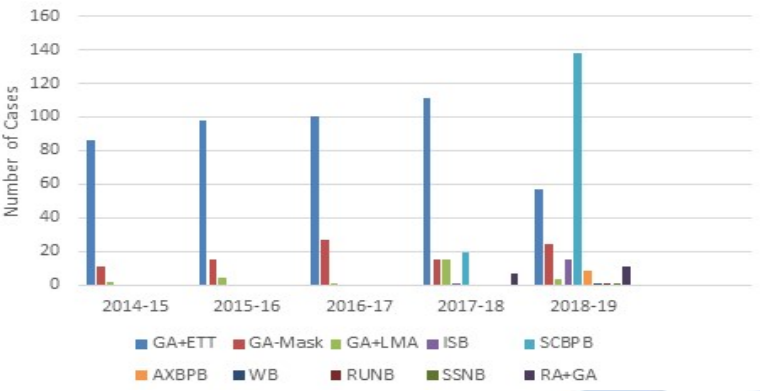

Figure 3: Distribtion of Type of Anaesthesia

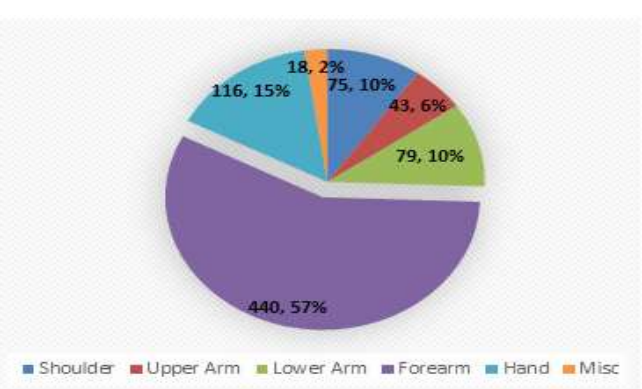

Figure 2: Distribution of Cases

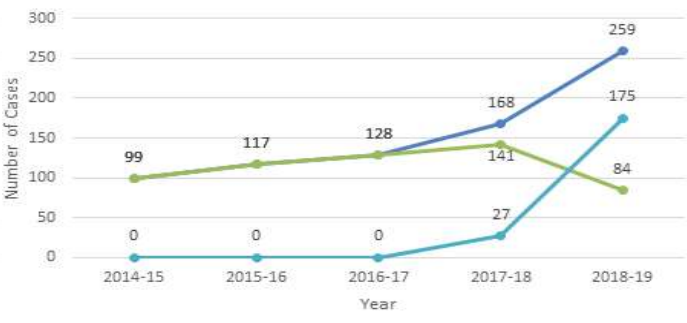

$\rightarrow$ Total $\rightarrow$ GA $\rightarrow R A$

Figure 4: Technique of Anaesthesia and Number of Cases

Table 1: Different Techniques of GA and RA practiced

\begin{tabular}{|c|c|c|c|c|c|c|c|c|c|c|c|}
\hline \multirow{2}{*}{ Year } & \multirow{2}{*}{ Total } & \multicolumn{3}{|c|}{ General Anaesthesia } & \multicolumn{7}{|c|}{ Regional Anaesthesia } \\
\hline & & GA+ETT & GA-Mask & GA+LMA & ISB & SCBPB & AXBPB & WB & RUNB & SSNB & RA+GA \\
\hline 2014-15 & 99 & 86 & 11 & 2 & & & & & & & \\
\hline $2015-16$ & 117 & 98 & 15 & 4 & & & & & & & \\
\hline 2016-17 & 128 & 100 & 27 & 1 & & & & & & & \\
\hline 2017-18 & 168 & 111 & 15 & 15 & 1 & 19 & & & & & 7 \\
\hline 2018-19 & 259 & 57 & 24 & 3 & 15 & 138 & 8 & 1 & 1 & 1 & 11 \\
\hline
\end{tabular}

Table 2: Trend of Anaesthesia Technique

\begin{tabular}{ccccc}
\hline year & Total & $\begin{array}{c}\text { General } \\
\text { Anaesthesia }\end{array}$ & $\begin{array}{c}\text { Regional } \\
\text { Anaesthesia }\end{array}$ & p-value \\
\hline $2014-15$ & 99 & 99 & 0 & - \\
$2015-16$ & 117 & $(100 \%)$ & & - \\
$2016-17$ & 128 & $(100 \%)$ & 0 & - \\
$2017-18$ & 168 & 128 & 0 & $<0.001$ \\
& & $(100 \%)$ & 27 & $<0.001$ \\
\hline
\end{tabular}

\section{DISCUSSION}

Nerve block anaesthesia being cheaper than GA has many advantages such as anaesthesia targeted at the operative site, excellent postoperative pain relief, decreased opioid use and reduced recovery time ${ }^{[16,17]}$. Obasuyi BI et al reported a predominant use of GA (84\%) for upper extremity surgeries, which is comparable with our observation made in the initial three years of the study, wherein GA (100\%) was the only technique of choice, but on the contrary, in the last two years of our study the practice of RA was significantly high $(16.07 \%$ and $67.56 \%$ respectively) ${ }^{[18]}$. Patel AA et al reported in their study that of all the upper limb surgeries, $20 \%$ are performed under peripheral nerve block and $80 \%$ are performed under general anaesthesia, which is comparable with our observation made in the year 2017- 
18 with GA $83.93 \%$, RA $16.1 \%$ of which 7 (4.2\%) patients received RA combined with GA ${ }^{[19]}$. But the same is not comparable with our observation made in the year $2018-19$, wherein $67.56 \%$ were provided RA and only $32.43 \%$ patients were provided GA. This may be due to their multicentic and total study duration data analysis effect and availability of equipment can be an added issue. Schnittger T et al and Rukewe A et al reported low utilisation of regional anaesthesia and peripheral nerve blocks and have blamed on deficiencies of training, equipments and drugs, which is comparable with our observation of the initial three years ${ }^{[20,21]}$.In our study, it is observed that the annual total number of upper extremity surgical cases have steadily increased from 99 patients in the year 2014-15 to 259 patients in the year 2018-19. This may be ascribed to progressive availability of better services and upgradation of our centre, compounded by increase in population. Our study showed that both GA and RA provided effective surgical anaesthesia for upper extremity surgical procedures, irrespective of the location or complexity of surgery for all the 771 patients studied. Regional anaesthesia when used as a sole technique or in conjunction with GA proved adequate for surgery in our study population. In our study, all the patients in the first 3 years of the study period received GA, and none were provided RA. In the year $2017-18$, the usage of RA rose to $16.07 \%$ from $0 \%$ in the preceding 3 years, and in the year 2018-19, it further increased to $67.56 \%$, thus indicating a significant change in the practice of anaesthetic techniques for upper extremity surgeries. This change in the trend of practice of anaesthetic techniques may be ascribed to the availability of necessary resource person, training, drugs and equipments during the period of beginning and progressive increase in the usage of RA. Availability of ultrasound with proper training promotes the practice of regional anaesthesia safely for surgeries of upper extremities.

\section{CONCLUSION:}

The use of RA for upper extremity surgery is steadily increasing in the recent years which is a positive development in a resource limited setting, and the most commonly RA technique employed is USG-guided Supraclavicular Brachial Plexus Block. There is a need to explore and encourage the use of other forms of regional anaesthesia also for upper extremity surgery.

\section{REFERENCES}

1. Hadzic A, Arliss J, Kerimoglu B, Karaca PE, Yufa M, et al. A Comparison of Infraclavicular Nerve Block versus General Anesthesia for Hand and Wrist Day-case Surgeries. Anesthesiology 2004; 101: 127-32.

2. Mariano ER, Chu LF, Peinado CR, Mazzei WJ. Anesthesia-controlled time and turnover time for ambulatory upper extremity surgery performed with regional versus general anesthesia. J Clin Anesth 2009; 21: 253-7.

3. Perlas A, Chan VWS, Simons M. Brachial plexus examination and localization using ultrasound and electrical stimulation: a volunteer study. Anesthesiology 2003; 99(2): 429-35.

4. D'Alessio JG, Rosenblum M, Shea KP, Freitas DG. A retrospective comparison of interscalene block and general anesthesia for ambulatory surgery shoulder arthroscopy. Reg Anesth Pain Med 1995; 20(1): 62-8.

5. Brown AR, Weiss R, Greenberg C, Flatow EL, and Bigliani LU. Interscalene block for shoulder arthroscopy: comparison with general anesthesia. Arthroscopy 1993; 9(3): 295-300.

6. Sia S, Lepri A, Ponzecchi P. Axillary brachial plexus block using peripheral nerve stimulator: a comparison between double- and triple-injection techniques. Reg Anesth Pain Med 2001; 26: 499-503.

7. 7.Greenblatt GM, Denson JS. Needle nerve stimulatorlocator: nerve blocks with a new instrument for location of nerves. Anaesth Analg 1962; 41: 599-602.

8. Sia S, Bartoli M, Lepri A, Marchini O, Ponsecchi P. Multiple-injection axillary brachial plexus block: a comparison of two methods of nerve localization-nerve stimulation versus paresthesia. Anesth Analg 2000; 91: 647-51.

9. Winnie AP. Does the transarterial technique of axillary block provide a higher success rate and lower complication rate than a paresthesia technique? Reg Anesth. 1995; 20: 482-5.

10. Baranowski AP, Pither CE. A comparison of three methods of axillary brachial plexus anesthesia. Anesthesia 1990; 45: 362-5.

11. Liu SS, Zayas VM, Gordon MA et al. A prospective randomized controlled trial comparing ultrasound versus nerve stimulator guidance for interscalene block for ambulatory shoulder surgery for postoperative neurological symptoms. Anesth Analg 2009; 109(1): 26571.

12. Hopkins PM. Ultrasound guidance as a gold standard in regional anaesthesia. Br J Anaesth 2007; 98(3): 299-301.

13. McCartney CJ, Lin L, Shastri U. Evidence basis for the use of ultrasound for upper-extremity blocks. Reg Anesth Pain Med. 2010; 35(2 suppl): S10-S15.

14. Domingo-Triado V, Selfa S, Martinez F et al. Ultrasound guidance for lateral midfemoral sciatic nerve block: a prospective, comparative, randomized study. Anesth Analg 2007; 104(5): 1270-74.

15. Marhofer P, Sitzwohl C, Greher M, Kapral S. Ultrasound guidance for infraclavicular brachial plexus anaesthesia in children. Anesthesia 2004; 59(7): 642-6.

16. Maga JM, Cooper L, Gebhard RE. Outpatient regional anesthesia for upper extremity surgery update (2005 to present) distal to shoulder. Int Anesthesiol Clin 2012; 50: 47-55.

17. Ilfeld BM, Wright TW, Enneking FK, Vandenborne K. Total elbow arthroplasty as an outpatient procedure using a continuous infraclavicular nerve block at home: a prospective case report. Reg Anesth Pain Med 2006; 31 : 172-6. 
18. Obasuyi BI, Alagbe-Briggs OT, Echem RC. Choice of anaesthesia for orthopaedic surgeries in a developing country: How appropriate? Journal of Medicine and Medical Sciences 2013; 4: 101-6.

19. Patel AA, Buller LT, Fleming ME, Chen DL, Owens PW, Askari M. National trends in ambulatory surgery for upper extremity fractures: a 10-year analysis of the US
National Survey of Ambulatory Surgery. Hand N Y N. 2015; 10(2): 254-9.

20. Schnittger T. Regional anaesthesia in developing countries. Anaesthesia 2007; 62 (suppl 1): 44-7.

21. Rukewe A, Fatiregun A. The use of regional anesthesia by anesthesiologists in Nigeria. Anesth Analg 2010; 110: 243-4.

Source of Support: None Declared Conflict of Interest: None Declared 